\title{
Potential of Serum and Urinary Matrix Metalloproteinase-9 Levels for the Early Detection of Renal Involvement in Children With Henoch-Schönlein Purpura
}

\author{
Meltem Erol, ${ }^{1,}$ Ozgul Yigit,, Mehmet Tasdemir, ${ }^{2}$ Ozlem Bostan Gayret, ${ }^{1}$ Ovgu Buke, ${ }^{1}$ Aysegul Gunes, ${ }^{3}$ \\ Sahin Hamilcikan, ${ }^{1}$ and Ozgur Kasapcopur ${ }^{4}$ \\ ${ }^{1}$ Department of Pediatrics, Bagcilar Training and Research Hospital, Istanbul, Turkey \\ ${ }^{2}$ Department of Pediatric Nephology, Koc University Hospital, Istanbul, Turkey \\ ${ }^{3}$ Department of Biochemistry, Bagcilar Training and Research Hospital, Istanbul, Turkey \\ ${ }^{4}$ Department of Pediatric Rheumatology, Cerrahpasa Medical Faculty, Istanbul University, Istanbul, Turkey \\ "Corresponding author: Meltem Erol, Department of Pediatrics, Bagcilar Training and Research Hospital, Istanbul, Turkey. Tel: +90-5324578397, Fax: +90-2124404000, E-mail: \\ drmeltemerol@yahoo.com
}

Received 2016 March 25; Revised 2016 April 19; Accepted 2016 May 10.

\begin{abstract}
Background: Matrix metalloproteinase-9 (MMP-9) is an enzyme implicated in the pathogenesis of renal diseases. Renal involvement is the principal cause of morbidity and mortality in children with Henoch-Schönlein purpura (HSP).

Objectives: The aim of this study was to evaluate whether serum and urinary MMP-9 levels are associated with renal involvement in HSP

Patients and Methods: We evaluated 40 children with HSP (patient group) and 27 healthy volunteer children (control group). The patient group was divided into two subgroups based on the presence or absence of nephritis. Nephritis was defined as the existence of hematuria and/or proteinuria. All anthropometric data, physical examination findings, blood pressure, and laboratory parameters were recorded. The serum and urine samples were analyzed to determine the MMP-9 levels three days after the initial phase of the disease.

Results: The mean age was $7.65 \pm 3.41$ (range $2-16$ ) years in the patient group and $9.52 \pm 3.91$ (range $2-16$ ) years in the control group. Henoch-Schonlein purpura nephritis (HSPN) was identified in eight patients. There was no significant difference in the serum MMP9 levels between the HSPN subgroup and the controls $(\mathrm{P}>0.05)$. However, there were significant differences in the urinary MMP-9 levels between the HSP subgroup and the control group $(\mathrm{P}<0.05)$, with the urinary MMP-9 levels being significantly higher in patients in the HSP subgroup $(\mathrm{P}=0.001)$. Further, the urinary MMP-9 levels were significantly higher in the patients with nephritis than in the patients without nephritis $(\mathrm{P}=0.001)$ and the controls $(\mathrm{P}=0.001)$. The optimal cut-off point (sensitivity; specificity) of the urinary MMP-9 level for the diagnosis of HSPN was $94.7 \mathrm{pg} / \mathrm{mL}$.

Conclusions: The levels of MMP-9 in the urine were remarkably high in patients with HSPN. This non-invasive marker may therefore be an important indicator for the early diagnosis of nephritis in children with HSP.
\end{abstract}

Keywords: Henoch-Schönlein Purpura, Henoch-Schönlein Purpura Nephritis, Matrix Metalloproteinase-9

\section{Background}

Henoch-Schönlein purpura (HSP) is one of the most common forms of vasculitis that affects the small blood vessels in children $(1,2)$. This form of leukocytoclastic vasculitis is associated with IgA-mediated immune deposits and it predominantly affects the skin, gastrointestinal tract, joints, and kidneys (3). Although HSP can be self-limiting, it can sometimes lead to serious complications. Renal involvement is the most important and lifethreatening complication of HSP. In the pediatric population, HSP is usually known as Henoch-Schönlein purpura nephritis (HSPN) when it affects the kidneys. Affected children have a 40\% risk of developing nephritis within 4 - 6 weeks of the initial presentation (4). HSPN that is characterized by the presence of hematuria alone or by different levels of proteinuria $(5,6)$ is usually a benign condition; however, it can also lead to renal failure (4).

The matrix metalloproteinases (MMPs) are a family of zinc-dependent proteinases and they are the main promoters of extracellular matrix (ECM) degradation. The MMP family plays a major role in ECM synthesis and breakdown in healthy kidney glomeruli (7). The imbalance between ECM synthesis and breakdown, as well as a deteriorating construction matrix, are important for the progression to glomerular disease (8).

Currently, the cause of HSPN remains unclear (2). However, glomerular mesangial cell proliferation and mesan- 
gial matrix accumulation are thought to be responsible for the development of renal involvement (7). Early diagnostic criteria are still being investigated for the diagnosis of HSPN. As MMP-9 has been reported to be the most important proteolytic enzyme involved in ECM remodeling, it is thought to be responsible for renal impairment (9).

Previous studies have reported changes in the serum and urinary MMP-9 levels in patients with $\operatorname{HSPN}(7,10-18)$. However, only a limited number of studies have so far been conducted on this subject.

\section{Objectives}

In the present study, we evaluated the role of MMP-9 in renal impairment by measuring the serum and urinary MMP-9 levels of children with HSP.

\section{Patients and Methods}

This study was conducted in the Department of Pediatrics at Bagcilar Training and research hospital in Istanbul, Turkey, between January 2014 and December 2015. Forty HSP patients and 27 healthy children were included in the study. HSP was diagnosed in patients with nonthrombocytopenic palpable purpura on the extensor aspects of the legs or on the buttocks (mandatory criterion) in the presence of at least one of the following four features: diffuse abdominal pain, any biopsy showing a predominant IgA deposition, arthritis or arthralgia, or renal involvement (any hematuria and/or proteinuria)(19). Children who required regular treatment for a chronic disease and those with urinary tract or systemic infections were excluded from the study. The control group was made up of healthy volunteers from the same age group. All anthropometric data and physical examination findings related to the control group were normal.

All anthropometric data, physical examination findings, blood pressure, biochemistry, and coagulation test results were recorded. Serum and urine samples were obtained from patients admitted to our clinics with a purpuric rash and then diagnosed with HSP within the first three days of admission. At baseline, none of the patients had renal involvement. All serum and urine samples were centrifuged for 5 minutes before being stored at $-20^{\circ} \mathrm{C}$ prior to use. The overall length of follow-up for all patients was one year.

In eight patients, renal involvement developed during the 3rd week of the follow-up period. Renal involvement is indicated by abnormal urinalysis results in HSP patients and it is categorized into three types: normal levels of proteinuria (proteinuria $<0.5 \mathrm{~g} / \mathrm{L}$ ) and hematuria $(\geq 5 \mathrm{red}$ blood cells per high-power microscopic field), low-grade proteinuria $(<1 \mathrm{~g} / \mathrm{L})$ and/or hematuria $(\geq 5$ red blood cells per high-power microscopic field), or heavy proteinuria $(\geq$ $1 \mathrm{~g} / \mathrm{L}$ ) and/or hematuria ( $\geq 5$ red blood cells per high-power microscopic field) $(5,6)$.

The Human MMP-9 ELISA kit (Ab100610, Abcam, USA) was used for the quantitative measurement of MMP-9 in the serum and urine samples. The samples and standards were added to the appropriate wells of a plate pre-coated with an anti-human monoclonal antibody prior to incubation. Then, biotin was added to the wells and combined with Streptavidin-HRP to form immune complexes. The incubation and washing steps were performed to remove the uncombined enzyme. Chromogen solutions A and B were added, thereby changing the color of the liquid to blue. The effect of the acid changed the color to yellow. The optical density was read on a standard automated plate reader at $450 \mathrm{~nm}$ (Perkin Elmer, 1420 Victor3). The detection range of the kit was between 24.69 and $12.000 \mathrm{pg} / \mathrm{mL}$.

The study was approved by the local ethics committee (2014 - 244), and informed consent was obtained from all children and/or their parents.

\subsection{Statistics}

All statistical descriptions and hypothesis tests were analyzed using SPSS version 23 and MedCalc 16.2 software. The explanatory statistics are given as the mean \pm standard deviation for the continuous variables and as the frequency and percentage for the discrete variables. The conformity of the continuous variables with the normal distribution was evaluated using the Kolmogorov-Smirnov test. Two independent sample t-tests were used to compare the variables that were in conformity with the normal distribution, while the Mann-Whitney U test was used to compare the variables that were not in conformity with the normal distribution. A ROC analysis was conducted to evaluate whether the serum and urinary MMP-9 values of the patient group were distinct from those of the control group, and the area under the curve was assessed. $\mathrm{P}<0.05$ was accepted as statistically significant.

\section{Results}

The mean age was $7.65 \pm 3.41$ years in the patient group and $8.67 \pm 3.78$ years in the control group, while the male/female ratio was $23 / 17$ in the patient group and $16 / 11$ in the control group. There were no significant differences in terms of age and gender between the patient and control groups. The patient height and weight percentiles were 25 - 50. All patients had a polymorphic rash with petechiae on the lower extremities, buttocks, and feet. 
Seven patients had swollen joints, particularly the knees and ankles, while 12 patients had abdominal pain due to HSP. This acute episode lasted for seven days. Skin biopsies were performed in three cases presenting with purpura but no renal involvement, abdominal pain, or arthritis. In these three patients, the skin biopsies were consistent with leukocytoclastic vasculitis and an immunofluorescence examination revealed immunoglobulin A deposits.

HSPN was identified in eight patients. Only two patients with renal involvement had a blood pressure above the 95th percentile; the other patients exhibited normal blood pressure. All patients had normal kidney function and platelet levels. Coagulation system tests revealed that the fibrinogen and activated partial thromboplastin time (aPTT) concentrations and the prothrombin time were normal. Ultrasonography of the abdomen revealed the kidneys to be of normal size and echogenicity.

\subsection{Comparison of HSP Patients Without Renal Involvement and the Control Group}

There was no significant difference between the serum MMP-9 levels of the HSP patients without renal involvement and those of the control group. However, the urinary MMP-9 levels in these patients were significantly higher than those of the control group $(\mathrm{P}<0.05$; Table 1$)$.

\subsection{Comparison of Patients with HSPN and the Control Group}

There was no significant difference between the serum MMP-9 levels of patients with renal involvement and those of the control group $(\mathrm{P}>0.05)$. Yet, the urinary MMP-9 levels of the patients with HSPN were significantly higher when compared to those of the control group $(\mathrm{P}=0.001$; Table 2).

A ROC analysis was performed to obtain the area under the curve and the optimal cut-off point for urinary MMP-9 for the diagnosis of HSPN. The optimal cut-off point (sensitivity; specificity) for the urinary MMP-9 level was found to be $94.7 \mathrm{pg} / \mathrm{mL}$. Thus, patients with urinary MMP-9 levels higher than 94.7 can be evaluated as patients with renal involvement $(\mathrm{P}<0.05$; Figure 1$)$.

\subsection{Comparison of the Three Groups}

The urinary MMP-9 level of the patients with HSPN was significantly higher than the levels of the HSP patients without renal involvement and the control group $(\mathrm{P}<$ 0.005; Table 3).

\section{Discussion}

One of the most serious complications that can develop during the course of HSP is renal involvement, which

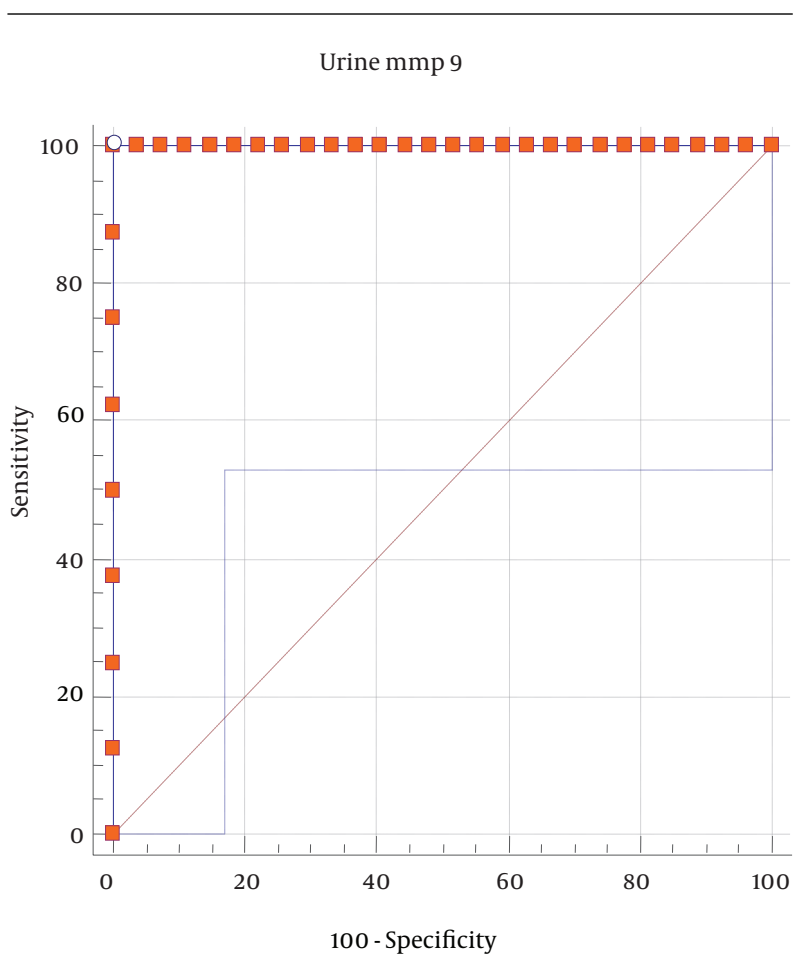

Figure 1. Receiver-Operator Curves of Urinary Matrix Metalloproteinase-9 for the Diagnosis of HSPN

is evaluated as HSPN. Non-invasive biomarkers that can detect renal involvement during the early stage and that may develop over the course of HSP have been investigated; however, the studies previously conducted on this issue have proved inadequate. The matrix metalloproteinases are known to play a role in renal involvement during inflammatory events. Due to this involvement, the present study was conducted to determine the possibility of using MMP-9 for the early detection of renal damage in patients with HSP.

Under normal physiological conditions, both MMP-9 activation and secretion with the matrix degradation protein are very low $(20,21)$. MMP-9 becomes active during inflammatory events, leading to the migration of leukocytes throughout the basal membrane and extracellular matrix degradation due to inflammatory precursor cytokine release from the extracellular matrix (22). Danilewicz et al. (23) reported that the renal damage in HSP patients with nephritis and IgA nephropathy showed similarities. The changes in glomerular cell proliferation and the ECM content and composition led to sclerosis and fibrosis in the glomeruli, which could progress to end-stage renal damage. The authors also reported that MMP-9 was responsible for the event that initiated EMC degradation in the 
Table 1. Comparison of the Serum and Urinary MMP-9 Levels of the HSP Group and the Control Group

\begin{tabular}{|c|c|c|c|}
\hline MMP-9 $(\mathbf{p g} / \mathrm{mL})$ & $\mathbf{N}$ & Mean \pm SD & PValue $^{\mathrm{a}}$ \\
\hline \multicolumn{4}{|l|}{ Serum } \\
\hline Control & 27 & $4917.1 \pm 2340.1$ & \\
\hline HSP & 32 & $5358.3 \pm 1393.9$ & 0.903 \\
\hline \multicolumn{4}{|l|}{ Urine } \\
\hline Control & 27 & $28.2 \pm 16.8$ & \\
\hline HSP & 32 & $71.8 \pm 98.4$ & 0.001 \\
\hline
\end{tabular}

${ }^{\mathrm{a}} \mathrm{P}<0.05$ is significant.

Table 2. Comparison of the Serum and Urinary MMP-9 Levels of the HSPN Group and the Control Group

\begin{tabular}{|c|c|c|c|}
\hline MMP-9 (pg/mL) & $\mathbf{N}$ & Mean \pm SD & PValue $^{\mathrm{a}}$ \\
\hline \multicolumn{4}{|l|}{ Serum } \\
\hline Control & 27 & $4917.1 \pm 2340.1$ & \\
\hline HSPN & 8 & $6101.5 \pm 809.7$ & 0.550 \\
\hline \multicolumn{4}{|l|}{ Urine } \\
\hline Control & 27 & $28.2 \pm 16.8$ & \\
\hline HSPN & 8 & $4307.9 \pm 2796.5$ & 0.011 \\
\hline
\end{tabular}

${ }^{\mathrm{a}} \mathrm{P}<0.05$ is significant.

Table 3. Comparison of Urinary MMP-9 Levels of the Three Groups ${ }^{\mathrm{a}}$

\begin{tabular}{lccc}
\hline Urine MMP-9 $(\mathbf{p g} / \mathbf{m L})$ & Control & HSP & HSPN \\
\hline $\mathbf{N}$ & 27 & 32 & 8 \\
Mean \pm SD & $28.2 \pm 16.8$ & $71.8 \pm 98.4$ & 40 \\
P value & & $0.011^{{ }^{*} \mathrm{~A}}$ & $0.001^{{ }^{\mathrm{B}}}$ \\
\hline
\end{tabular}

${ }^{\mathrm{a}}$ The Mann-Whitney $\mathrm{U}$ test was used as a post hoc test to identify group differences, and the P values were interpreted with the Bonferroni correction. $\mathrm{P}<0.05$ was significant. $\mathrm{P}^{* \mathrm{~A}}$ is the comparison between patients without renal involvement and the control group, $\mathrm{P}^{{ }^{\mathrm{B}}}$ is the comparison between patients with renal involvement and the control group, and $\mathrm{P}^{*} \mathrm{C}$ is the comparison between HSP patients without renal involvement and those with renal involvement.

glomeruli in both diseases.

The mechanism of serum and urinary MMP-9 elevation in patients with HSP and HSPN is not yet known, although different opinions on the matter do exist. Abnormal EMC remodeling is responsible for the glomerulus damage in HSPN (23). One possible explanation is the excretion of MMP-9 resulting in increased levels in the serum and urine as a result of inflammation in patients with HSPN (24). The excretion of MMP-9 in the urine increases concomitantly with its increase in the serum. In a study performed by Shin et al. (25) investigating the MMP-9 gene expression profiles of children with HSP, the serum MMP level during the acute phase was significantly higher than that of the control group.

In a meta-analysis evaluating eight studies, the serum
MMP-9 level was reported to be a risk factor for the development of HSPN (26). All eight studies were conducted in a Chinese population, with seven published in Chinese and only one published in English (7). In the latter (7), both the urinary and serum MMP-9 levels were higher in children with renal involvement when compared to children with HSP but without renal involvement and children in the control group. Moreover, the serum MMP-9 level was reported to exhibit a five-fold increase in patients with HSPN when compared to the control group's serum MMP-9 level. The authors found that the optimal cut-off point (sensitivity; specificity) for the serum MMP-9 level for the diagnosis of HSPN was $179.79 \mathrm{mg} / \mathrm{L}(0.96 ; 0.88)$ (7). Furthermore, serum MMP-9 was reported to possibly predict the onset of HSPN in children. 
In our study, the serum MMP-9 level in HSP patients with and without renal involvement did not differ from that of the control group. Thus, the results of our study were not consistent with those of previous studies. In a study conducted by Zou et al. (27), the serum MMP level in samples collected from acute phase HSP patients without renal involvement was significantly higher than the level found in samples collected during the healing phase, while the serum MMP-9 level of some HSP patients was lower than the levels found in the control group. This situation was explained by the finding that the MMP-9 level could increase in cases other than HSP (27). In our study, only healthy children with no acute or chronic illness were included; hence, the high MMP-9 levels detected in the control group were not correlated with any other cause.

Another possible explanation for the increased MMP9 level in the urine and serum is that the increase in the MMP-9 level during inflammation deteriorates membrane integrity and permeability, leading to damage to the glomerular basal membrane and thereby increasing the MMP-9 level in the urine (28).

Previous studies reported that the urinary MMP-9 level was increased in HSP patients with renal involvement (7, 10-12). In a meta-analysis evaluating four studies, the urinary MMP-9 level was found to be increased in patients with HSPN and hence it could be used as an early indicator (26). In this study, the urinary MMP-9 level of the patients with HSPN was higher than that of the patients without renal involvement and the control group. The results of our study are therefore consistent with the results of other studies reported to date. Under normal conditions, a robust glomerulus basal membrane does not allow the passage of these small MMP-9 molecules (23). The urinary MMP-9 level in both the HSP and HSPN patients was high during the early period of the respective disease in this study, which might indicate that the glomerulus damage started early. A limitation of this study is the insufficient number of patients with HSPN.

\subsection{Conclusion}

The urinary MMP-9 level could be a useful indicator of renal damage during the acute phase in patients with HSPN. However, further studies are needed to support this hypothesis.

\section{Acknowledgments}

The authors wish to thank Bagcilar training and research hospital. This study was reviewed and approved by the review board of Bagcilar training and research hospital.

\section{Footnote}

Funding/Support: This study was supported by the educational planning coordination committee of Bagcilar training and research hospital

\section{References}

1. Yi ZW, Fang XL, Wu XC, He XJ, He QN, Dang XQ, et al. Role of PAX2 gene polymorphisms in Henoch-Schonlein purpura nephritis. Nephrology (Carlton). 2006;11(1):42-8. doi: 10.1111/j.1440-1797.2006.00537.x. [PubMed: 16509931].

2. Lau KK, Suzuki H, Novak J, Wyatt RJ. Pathogenesis of HenochSchonlein purpura nephritis. Pediatr Nephrol. 2010;25(1):19-26. doi: 10.1007/s00467-009-1230-x. [PubMed: 19526254].

3. Davin JC, Weening JJ. Henoch-Schonlein purpura nephritis: an update. Eur J Pediatr. 2001;160(12):689-95. [PubMed: 11795675].

4. Kawasaki Y, Ono A, Ohara S, Suzuki Y, Suyama K, Suzuki J, et al. HenochSchonlein purpura nephritis in childhood: pathogenesis, prognostic factors and treatment. Fukushima J Med Sci. 2013;59(1):15-26. [PubMed: 23842510].

5. de Almeida JL, Campos LM, Paim LB, Leone C, Koch VH, Silva CA. Renal involvement in Henoch-Schonlein purpura: a multivariate analysis of initial prognostic factors. J Pediatr (Rio J). 2007;83(3):259-66. doi: 10.2223/JPED.1638. [PubMed: 17551657].

6. Hung SH, Yang YH, Lee CC, Wang LC, Lin YT, Chiang BL. Clinical predictors of self-limited urinalysis abnormality in childhood HenochSchonlein purpura nephritis. Acta Paediatr. 2006;95(3):306-11. doi: 10.1080/08035250500421584. [PubMed: 16497641].

7. Qin YH, Zhou TB, Lei FY, Huang WF, Zhao YJ, Lin FQ, et al. Cutoff values for serum matrix metalloproteinase-9: is there a threshold to predict renal involvement for Henoch-Schonlein purpura in children?. Nephrology (Carlton). 2011;16(1):93-9. doi: 10.1111/j.14401797.2010.01360.x. [PubMed: 21175984].

8. Lenz O, Elliot SJ, Stetler-Stevenson WG. Matrix metalloproteinases in renal development and disease. J Am Soc Nephrol. 2000;11(3):574-81. [PubMed: 10703682].

9. Catania JM, Chen G, Parrish AR. Role of matrix metalloproteinases in renal pathophysiologies. Am J Physiol Renal Physiol. 2007;292(3):F90511. doi: 10.1152/ajprenal.00421.2006. [PubMed: 17190907].

10. Ma H, Lin H, Ma G. Urinary levels of collagen IV and MMP-9 and their clinical significances in children with Henoch-Schonlein purpura. Med Innov Res. 2008;5:8-9.

11. Wang SM, Qi X, Pan T, Xu L. The Relationship between Urinary Microprotein and Metalloproteinase-9 Expression in Children with Nephritis of Schonle-Henoch Purpura. J Hubei Univ Med. 2010;29:515-7.

12. Li C, Chen Q, Zhang Y, Wang M, Ke P. Changes and clinical significance of matrix metalloproteinase-9 in children with Henoch-Schonlein purpura nephritis. Guangdong Med J. 2011;32:2945-6.

13. Zhu QY, Jiang YH, Liu JD, Sun Q. Significance of Changes of Serum Levels and Ratios of Matrix Nephritis Metalloproteinase-2,-9 and Tissue Inhibitor of Metalloproteinase-1 in Children with Henoch-Schonlein Purpura Nephristis. J Appl Clin Pediatr. 2007;22(5):343-4.

14. Caim W, Li A, Chen S, Li C, Cui H. Clinical significance of the change of matrix metalloproteinase-9 children with Henoch-Schönlein purpura nephritis. Shandong Med J. 2007;47:42-3.

15. Wang YB AS. Matrix metalloproteinase 2, 9 and its tissue inhibitor 1 expression and their significance in the Henoch-Schönlein purpura nephritis. Harbin Med J. 2009;29:13-4.

16. Lu Y. The clinical significance of the detection of matrix metalloproteinase 9 in children with Henoch-Schönlein purpura nephritis. Mod Pract Med. 2010;22:1178-80. 
17. Yu Z, Peng X. The changes and clinical significance of IL-6, IL-8, MMP9 in serum of children with Henoch-Schönlein purpura nephritis. $T$ Hebei Med. 2008;14:573-6.

18. Geng XH, Zhang H. Relationship between Matrix Metalloproteinase Level and Kidney Damage in Children with Henoch-Schonlein Purpura Nephritis. J Henan Univ Sci Tech (Med Sci). 2011;29:100-2.

19. Ozen S, Ruperto N, Dillon MJ, Bagga A, Barron K, Davin JC, et al. EULAR/PReS endorsed consensus criteria for the classification of childhood vasculitides. Ann Rheum Dis. 2006;65(7):936-41. doi: 10.1136/ard.2005.046300. [PubMed: 16322081].

20. McLennan SV, Fisher E, Martell SY, Death AK, Williams PF, Lyons JG, et al. Effects of glucose on matrix metalloproteinase and plasmin activities in mesangial cells: possible role in diabetic nephropathy. Kidney Int Suppl. 2000;77:S81-7. [PubMed: 10997695].

21. Tveita A, Rekvig OP,Zykova SN. Glomerular matrix metalloproteinases and their regulators in the pathogenesis of lupus nephritis. Arthritis Res Ther. 2008;10(6):229. doi: 10.1186/ar2532. [PubMed: 19090960].

22. Sanders JS, van Goor H, Hanemaaijer R, Kallenberg CG, Stegeman CA. Renal expression of matrix metalloproteinases in human ANCA-associated glomerulonephritis. Nephrol Dial Transplant. 2004;19(6):1412-9. doi: 10.1093/ndt/gfh186. [PubMed: 15034162].

23. Danilewicz M, Wagrowska-Danilewicz M. Differential glomerular immunoexpression of matrix metalloproteinases MMP-2 and MMP-9 in idiopathic IgA nephropathy and Schoenlein-Henoch nephritis. Folia Histochem Cytobiol. 2010;48(1):63-7. doi: 10.2478/v10042-008-0086-4 [PubMed: 20529817].

24. Tashiro K, Koyanagi I, Ohara I, Ito T, Saitoh A, Horikoshi S, et al. Levels of urinary matrix metalloproteinase-9 (MMP-9) and renal injuries in patients with type 2 diabetic nephropathy. J Clin Lab Anal. 2004;18(3):206-10. doi:10.1002/jcla.20024. [PubMed: 15103687].

25. Shin JI, Song KS, Kim H, Cho NH, Kim J, Kim HS, et al. The gene expression profile of matrix metalloproteinases and their inhibitors in children with Henoch-Schonlein purpura. BrJ Dermatol. 2011;164(6):134855. doi: 10.1111/j.1365-2133.2011.10295.x. [PubMed: 21410660].

26. Zhou TB, Yin SS. Association of matrix metalloproteinase-9 level with the risk of renal involvement for Henoch-Schonlein purpura in children. Ren Fail. 2013;35(3):425-9. doi: 10.3109/0886022X.2012.757826. [PubMed: 23356642].

27. Zou CC, Zhao ZY, Tang LF, Liang L. Plasma levels of matrix metalloproteinase-9 in Henoch-Schonlein purpura. Scand J Rheumatol. 2006;35(1):52-5. doi: 10.1080/03009740510026940. [PubMed: 16467043].

28. Ebihara I, Nakamura T, Shimada N, Koide H. Increased plasma metalloproteinase-9 concentrations precede development of $\mathrm{mi}$ croalbuminuria in non-insulin-dependent diabetes mellitus. Am J Kidney Dis. 1998;32(4):544-50. 